\title{
Unruh Effect without Thermality
}

\author{
Raúl Carballo-Rubio, ${ }^{1, *}$ Luis J. Garay, ${ }^{2, \dagger}$ Eduardo Martín-Martínez, ${ }^{3,4, \$}$ and José de Ramón ${ }^{3, \S}$ \\ ${ }^{1}$ SISSA, International School for Advanced Studies, Via Bonomea 265, 34136 Trieste, Italy \\ and INFN Sezione di Trieste, Via Valerio 2, 34127 Trieste, Italy \\ ${ }^{2}$ Departamento de Física Teórica and IPARCOS, Universidad Complutense de Madrid, 28040 Madrid, Spain \\ and Instituto de Estructura de la Materia (IEM-CSIC), Serrano 121, 28006 Madrid, Spain \\ ${ }^{3}$ Institute for Quantum Computing, University of Waterloo, Waterloo, Ontario, N2L 3G1, \\ Canada and Department of Applied Mathematics, University of Waterloo, Waterloo, Ontario, N2L 3G1, Canada \\ ${ }^{4}$ Perimeter Institute for Theoretical Physics, Waterloo, Ontario, N2L 2Y5, Canada
}

(Received 1 April 2018; revised manuscript received 26 November 2018; published 26 July 2019)

\begin{abstract}
We show that uniformly accelerated detectors can display genuinely thermal features even if the KuboMartin-Schwinger (KMS) condition fails to hold. These features include satisfying thermal detailed balance and having a Planckian response identical to cases in which the KMS condition is satisfied. In this context, we discuss that satisfying the KMS condition for accelerated trajectories is just sufficient but not necessary for the Unruh effect to be present in a given quantum field theory. Furthermore, we extract the necessary and sufficient conditions for the response function of an accelerated detector to be thermal in the infinitely adiabatic limit. This analysis provides new insight about the interplay between the KMS condition and the Unruh effect, and a solid framework in which the robustness of the Unruh effect against deformations of quantum field theories (perhaps Lorentz-violating) can be answered unambiguously.
\end{abstract}

DOI: 10.1103/PhysRevLett.123.041601

Quantum field theory (QFT) is considered to be an effective theory that is valid outside the quantum gravity domain, typically defined in terms of a length scale $\ell$ [1]. This theoretical framework is therefore expected to become less precise as this scale is approached, perhaps eventually failing completely as a correct description of nature. In most cases, there is a hierarchy of scales that ensures that the predictions of QFT within its domain of validity are not contaminated by the ultraviolet physics below $\ell$. However, it is known that ultraviolet deformations of the structure of QFT can percolate into this domain of validity, spoiling the decoupling of scales [2-6]. When present, this phenomenon brings the possibility of testing theoretical frameworks which would be otherwise impossible (or extremely difficult) to probe. Examples of this behavior that have been recently discussed in the literature include the response of particle detectors along inertial trajectories in the framework of modified dispersion relations [7], polymer quantization [8-10], and in nonlocal field theories [11], or the transmission of information through nonlocal fields [12]. Determining the deformations that lead to this percolation, and finding the predictions which are affected, is of clear importance for quantum gravity phenomenology.

Here, we focus on a central prediction of QFT: the Unruh effect [13-15]. This well-known phenomenon [16] illustrates that the concept of particle is observer dependent in QFT, which is inextricably linked to black hole evaporation $[17,18]$. Aside from its importance on theoretical grounds, there are reasonable prospects for detecting this effect in the near future (see, e.g., Refs. [19-22]). In standard Lorentzinvariant QFT, the Unruh effect is defined as the perception of a thermal bath by uniformly accelerated observers that interact with a quantum field in its ground state [23]. The interaction of the observer with the field is modeled in terms of particle detectors [15,24-26], whose coupling to the field is characterized by their so-called response function [27]. The thermal behavior of the response function of uniformly accelerated detectors is guaranteed by the Kubo-Martin-Schwinger (KMS) condition [28-30] that characterizes thermal states. Hence, the KMS condition is sometimes taken as the definition of the Unruh effect. However, one can question whether the field state being KMS for accelerated observers is necessary for a detector to perceive the Unruh effect.

Indeed, as we make explicit below, large sets of deformations of QFT (including the introduction of a cutoff on spatial momenta) lead to violations of the KMS condition even in the vacuum state. There are two possible attitudes with respect to this observation. The first one is to assume directly that these deformations would erase any trace of the Unruh effect (see, for instance, Ref. [31] for a particular example in the framework of polymer quantization). The second one, put forward in this Letter, is admitting that the KMS condition is unnecessarily restrictive from a physical perspective. In the presence of deformations of QFT with typical length scale $\ell$, it is reasonable to expect that small deviations from an exact thermal behavior, involving this new scale, would appear. This broader set of scenarios 
cannot be characterized by the KMS condition, which will be generally violated even though the response function can display thermal features. This has been noticed before in particular scenarios involving cavities [32], specific curved geometries [33], or excited states in theories in which the ground state is KMS [34].

Following this intuition, here we systematically analyze and determine the minimal requirements that single out the scenarios in which the violations of the KMS condition are mild enough so that the Unruh effect is preserved. We first prove that the long-time response function of a uniformly accelerated detector interacting with fields that are invariant under spacetime translations and spatial rotations (but not necessarily Lorentz boosts) is reduced, in the adiabatic limit, to a single-variable integral of the sum of the residues of the poles of the Wightman function inside a horizontal strip of the complex plane. This general result permits us to calculate explicitly the response function in a generality of situations, allowing us to critically revise cases of particular deformations studied previously [31,35-42], and define in general terms the conditions for the preservation of the Unruh effect. Crucially, we find that the preservation of the Unruh effect is less restrictive than the KMS condition.

Deformations of the Wightman function.-A relativistic QFT is given by a Hilbert space $\mathbb{\square}$ of states and a set of unitary operators associated with the transformations in the Poincaré group. $\mathbb{W}$ has to contain a unique state $|0\rangle$ invariant under Poincaré transformations. Field operators $\phi(X)$ are operator-valued distributions acting on the space of test functions defined over $\mathbb{R}^{n}$. The two remaining conditions are energy positivity and locality (field operators commute on spacelike intervals).

Wightman functions contain the full information about a QFT satisfying the axioms above [43]. For our purposes here, it is enough to study the two-point Wightman function $\mathcal{W}\left(X^{\prime \prime}, X^{\prime}\right)=\left\langle 0\left|\phi\left(X^{\prime \prime}\right) \phi\left(X^{\prime}\right)\right| 0\right\rangle$, since the leading order detector response for any state is only a function of this quantity through the so-called response function:

$$
\begin{aligned}
\mathcal{F}(\Omega, \sigma)= & \frac{1}{\sigma} \int_{-\infty}^{\infty} d \tau^{\prime \prime} \int_{-\infty}^{\infty} d \tau^{\prime} \chi\left(\tau^{\prime \prime} / \sigma\right) \chi\left(\tau^{\prime} / \sigma\right) \\
& \times W\left(\tau^{\prime \prime}, \tau^{\prime}\right) e^{-i \Omega\left(\tau^{\prime \prime}-\tau^{\prime}\right)} .
\end{aligned}
$$

Here, $W\left(\tau^{\prime \prime}, \tau^{\prime}\right)$ is the pullback of the Wightman function $\mathcal{W}\left(X^{\prime \prime}, X^{\prime}\right)$ to a given trajectory in spacetime, $X(\tau)$. Equation (1) arises naturally in the study of the excitation and decay probabilities in the Unruh-DeWitt model of a detector interacting with the field $\phi(X)$ (see Ref. [44], for instance, for a detailed description), which assumes an interaction Hamiltonian $H_{I}(\tau)=\lambda \chi(\tau / \sigma) \mu(\tau) \phi(X(\tau))$, where $\mu(\tau)$ is the monopole moment operator of the detector and $\chi(\tau / \sigma) \in C^{\infty}(\mathbb{R})$ is a square-integrable switching function that controls the duration and the form of the window of time in which the interaction between the detector and the field takes place. This kind of switching is known as adiabatic [44,45], and it depends on a single width parameter $\sigma$ that provides a measure of the interaction time scale (so that the infinitely adiabatic limit $\sigma \rightarrow \infty$ corresponds to a detector switched on forever). The probabilities of excitation and decay are proportional to Eq. (1), with respectively positive and negative values of $\Omega$ (the energy gap of the detector is $|\Omega|$ ). However, the corresponding proportionality factors are independent of the properties of the QFT, and only depend on the properties of the detector (including its monopole moment) and the coupling $\lambda \in \mathbb{R}$ between the detector and the field $[27,46]$. Hence, the quotients of these probabilities, which are the quantities of interest in order to determine whether or not the response of the detector is thermal, are independent of these proportionality factors.

The Wightman function is a distribution, so that Eq. (1) would be meaningful only for suitable choices of the space of switching functions such as, for instance, functions with noncompact support that decay faster than any polynomial. For our purposes here, it will be enough to consider Gaussian switching functions normalized so that $\int_{-\infty}^{\infty} d y \chi(y)^{2}=1$, though other choices are possible. Hence, we can safely forget about the distributional nature of the Wightman function (and its deformations introduced below), and work with it as if it was a function, as long as we keep in mind that this quantity is always under the integral sign in Eq. (1).

On general grounds, the introduction of an additional length scale $\ell$ leads to deformations of the functional form of the Wightman function. These deformations encode the leading modifications arising from the particular ultraviolet completion chosen, or may just represent physical cutoffs. Let us make the following technical assumptions.

(1) There is an effective continuum flat description of spacetime in which the deformed Wightman function can be written as a function of the spacetime coordinates $X^{\mu}=(t, \boldsymbol{x})$.

(2) The functional form of the deformed Wightman function may break explicitly the invariance under Lorentz boosts, while keeping spacetime translations and spatial rotations as symmetries.

These are fairly general assumptions. For instance, condition (1) permits us to include in our analysis discrete or quantum-mechanical features of the spacetime structure. Condition (2), together with the transformation rules for the vacuum state and field operators, implies that the Wightman function is invariant under translations, hence being a function of the differences $\Delta t=t^{\prime \prime}-t^{\prime}$ and $\Delta \boldsymbol{x}=\boldsymbol{x}^{\prime \prime}-\boldsymbol{x}^{\prime}$. Without loss of generality, we can always write the Wightman function in the fairly general form:

$$
\mathcal{W}_{\ell}=\mathcal{W}_{\ell}(\Delta t, \Delta \boldsymbol{x})
$$

Infinitely adiabatic limit for uniformly accelerated observers.-The response function $\mathcal{F}_{\ell}(\Omega, \sigma)$ associated with a given deformation takes the same form as Eq. (1) 
but with $W\left(\tau^{\prime \prime}, \tau^{\prime}\right)$ replaced with $W_{\ell}\left(\tau^{\prime \prime}, \tau^{\prime}\right)$. In the following, we consider only trajectories with constant acceleration $a$ and Gaussian switching functions, and change the integration variables to $w=\tau^{\prime \prime}+\tau^{\prime}$ and $z=\tau^{\prime \prime}-\tau^{\prime}$. It is straightforward to check that the pullback of any Wightman function of the form provided in Eq. (2) depends on $z$ only through the combination $\sinh (a z / 2)$ [47], which implies that $W_{\ell}(w, z+4 \pi i / a)=W_{\ell}(w, z)$. To the best of our knowledge, the existence of this symmetry has not been noticed before. This allows using the methods of complex analysis to deal with the response function, choosing a rectangular integration contour in the complex plane (we are further assuming that the deformed Wightman function extends to a meromorphic function in an open subset enclosing this contour, which is satisfied by a large class of deformations, including all the examples discussed explicitly here and in previous literature). The integral in $z$ can then be reduced to a sum of residues [47] under the following falloff condition.

(3) The deformed Wightman function is polynomially bounded in $|\Delta t|$ and $|\Delta \boldsymbol{x}|$ when these absolute values tend to infinity.

The result of these manipulations is

$$
\begin{aligned}
\mathcal{F}_{\ell}(\Omega, \infty)= & \frac{i \sqrt{\pi}}{1-e^{4 \pi \Omega / a}} \\
& \times \lim _{\sigma \rightarrow \infty} \frac{1}{\sigma} \int_{-\infty}^{\infty} d w e^{-w^{2} / \sigma^{2}} \sum_{k \in I} \operatorname{Res}\left[f_{\ell, \sigma}(z), z_{k}\right],
\end{aligned}
$$

where $f_{\ell, \sigma}(z)=e^{-z^{2} / \sigma^{2}} W_{\ell}(w, z) e^{-i \Omega z}$ with the slight abuse of notation $W_{\ell}(w, z)=W_{\ell}\left(\tau^{\prime \prime}(w, z), \tau^{\prime}(w, z)\right)$. On the other hand, $\left\{z_{k}\right\}_{k \in I}$ is the (finite) set of poles in $z$ of the Wightman function [equivalently, $f_{\ell, \sigma}(z)$ ] on the horizontal strip of the complex plane $S \subset \mathbb{C}$ defined by $0 \leq \operatorname{Im}[z] \leq 4 \pi / a$.

The Wightman function is generally singular on the real axis in the coincidence limit. This is typically dealt with introducing a regulator $z+i \epsilon$. This can also be understood as an infinitesimal displacement of one of the proper times $\tau^{\prime}$ or $\tau^{\prime \prime}$ (more details in this regard are given later). This regulator is removed in the final expression for the response function after integration. We assume that all the real poles in $z$ are regularized in the same way. In deformations that are Lorentz breaking, additional real poles in $w$ can appear. In this case, the infinitesimal displacement of the proper times $\tau^{\prime}$ or $\tau^{\prime \prime}$ leads to $w \pm i \epsilon$. Physical results should not depend on the sign of the regulator in $w$.

Preservation of the Unruh effect.-Equation (3) determines the infinitely adiabatic limit of the response function for all the deformations satisfying the requirements (1)-(3). We have used it in order to calculate the response function in several examples. The results are compiled in Table I. Most importantly, we want to highlight that Eq. (3) can be exploited in order to extract the conditions that guarantee the preservation of the Unruh effect.

On general grounds, the Unruh effect is preserved if the response rate of the detector along uniformly accelerated trajectories has the right $\ell \rightarrow 0$ limit, namely, if $\lim _{\ell \rightarrow 0} \mathcal{F}_{\ell}(\Omega, \infty)=\mathcal{F}(\Omega, \infty)$. Importantly, this does not imply that the contributions depending on $\ell$ vanish in the $\sigma \rightarrow \infty$ limit, but rather that they appear in the adiabatic response as subleading corrections to the usual Unruh effect. In the infinitely adiabatic limit, the only possible dimensionless combinations of the physical quantities involved are $\ell a$ and $\ell \Omega$. Hence, if the condition above is satisfied, the $\ell=0$ expressions for the response functions are recovered up to small corrections when $\ell a \ll 1$ and $\ell \Omega \ll 1$. In other words, appreciable deviations from

TABLE I. Comparison of the KMS condition and the sufficient conditions for the preservation of the Unruh effect in the adiabatic limit (for $n=4$ spacetime dimensions). "Imaginary periodicity" refers to the symmetry $z \rightarrow z+2 \pi i / a$ of the Wightman function [Eq. (6)], which is not equivalent to the (less restrictive) symmetry $z \rightarrow z+4 \pi i / a$, which is central to our analysis and is satisfied by all the deformations studied in this Letter. Let us note that, for simplicity, all the examples in this table are of the form $\mathcal{W}_{\ell}(\Delta t, \Delta \boldsymbol{x})=\mathcal{W}_{0}(\Delta X)\left[1+\mathcal{D}_{\ell}(\Delta t, \Delta \boldsymbol{x})\right]$ for some choice of function

\begin{tabular}{|c|c|c|c|c|c|}
\hline \multirow{2}{*}{$\mathcal{D}_{\ell}(\Delta t, \Delta \boldsymbol{x})$} & \multicolumn{4}{|c|}{ KMS } & \multirow[t]{2}{*}{ Preservation } \\
\hline & Imaginary periodicity & Stationarity & Holomorphicity & Polynomial & \\
\hline$\ell^{2} /\left(\Delta X^{2}+\ell^{2}\right)[35,38]$ & $\checkmark$ & $\checkmark$ & $\checkmark$ & $\checkmark$ & $\checkmark$ \\
\hline$-\alpha \ell^{2} /\left(\Delta X^{2}+\ell^{2}\right), \alpha \in \mathbb{R}$ & $\checkmark$ & $\checkmark$ & $\checkmark$ & $\checkmark$ & $\checkmark$ \\
\hline$-\ell^{2} /\left(\Delta X^{2}-\ell^{2}\right)[38]$ & $\checkmark$ & $\checkmark$ & $x$ & $\checkmark$ & $x$ \\
\hline$-e^{-\Delta X^{2} / \ell^{2}}[37,48]$ & $\checkmark$ & $\checkmark$ & $\checkmark$ & $x$ & $?$ \\
\hline$\ell^{2} / \Delta t^{2}$ & $\checkmark$ & $x$ & $\checkmark$ & $\checkmark$ & $\sqrt{ }$ \\
\hline$\ell /(i \Delta t+\ell)$ & $x$ & $x$ & $x$ & $\checkmark$ & $\checkmark$ \\
\hline$i \ell \Delta t / \Delta X^{2}$ & $x$ & $x$ & $\checkmark$ & $x$ & $x$ \\
\hline$i \ell / \Delta t$ & $x$ & $x$ & $\checkmark$ & $\checkmark$ & $\checkmark$ \\
\hline
\end{tabular}
$\mathcal{D}_{\ell}(\Delta t, \Delta \boldsymbol{x})$. The question mark indicates a deformation that does not satisfy our condition (3). 
the Unruh effect would only exist for accelerations or frequencies that are of the same scale as the inverse of the parameter of the deformation $\ell$.

It is worth mentioning that the study of the finite-time response, and not only its adiabatic limit, is a source of rich phenomenology (see among others Refs. [11,12,49,50]). This finite-time response would be sensitive to the details of the switching, including additional physical scales that appear in nonadiabatic switching functions. These additional scales may form new dimensionless combinations with high-energy scales that may not be necessarily small. This is just a reminder of the following general statement: that two different deformations preserve the Unruh effect does not necessarily imply that all other possible observables will agree.

We can now use Eq. (3) in order to identify the necessary and sufficient conditions that guarantee the preservation of the Unruh effect. Let us define the set of poles $\left\{\bar{z}_{l}\right\}_{l \in J}$ that are obtained as a continuous deformation of the original set of poles $\left\{z_{m}^{0}\right\}_{m \in K}$ originally in $S \subset \mathbb{C}$, with the possible addition or splitting of poles. We can then define the following conditions.

(A) Local uniform convergence.-The integral along each of the contours $\gamma_{i}$ containing all the deformed poles that stem from each of the poles $z_{i}^{0}$ of the undeformed Wightman function, but not from other poles of the latter, recovers the undeformed contribution in the $\ell \rightarrow 0$ limit.

(B) All the poles $\left\{\bar{z}_{l}\right\}_{l \in J}$ must remain in the horizontal strip $S \subset \mathbb{C}$; namely, $\left\{z_{k}\right\}_{k \in I}=\left\{\bar{z}_{l}\right\}_{l \in J}$.

(C) The sum of the residues of the poles in $S \subset \mathbb{C}$ of the Wightman function in $z$, times $e^{-w^{2} / \sigma^{2}}$, must be integrable with respect to $w$ in the $\sigma \rightarrow \infty$ limit.

(A)-(C) above are sufficient, when holding simultaneously, in order to preserve the Unruh effect. (A) and (C) are necessary conditions, and also (B) unless the contribution from the poles escaping the horizontal strip $S \subset \mathbb{C}$ is trivial for $\sigma \rightarrow \infty$.

Let us sketch the proof of this statement. Condition (C) implies that the right-hand side of Eq. (3) is finite in the $\sigma \rightarrow \infty$ limit. On the other hand, condition (B) implies that all the deformed poles that stem from undeformed poles $z_{m}^{0}$ inside the horizontal strip $S \subset \mathbb{C}$ remain in $S$. Therefore, the corresponding residues are all taken into account in the right-hand side of Eq. (3). Finally, condition (A) ensures that the sum of these residues has the right $\ell \rightarrow 0$ limit.

Regarding the Kubo-Martin-Schwinger condition.-As mentioned previously, in Lorentz-invariant QFT the thermal behavior of the response function holds exactly under the KMS condition. In fact, it can be seen [44] that this condition is sufficient for (thermal) detailed balance to be satisfied,

$$
\mathcal{F}(-\Omega, \infty)=e^{2 \pi \Omega / a} \mathcal{F}(\Omega, \infty),
$$

which is the smoking gun of thermal behavior. However, we have devoted this Letter to the determination of the minimal requirements that single out the scenarios in which this violation is mild enough so that detailed balance is still satisfied, possibly up to small corrections. For completeness, in this section we discuss the interplay between these different conditions, and illustrate the general discussion with examples (see Table I).

First, let us clarify the operational definition of the KMS condition that we will be using [44,51]. In fact, the KMS condition is, more strictly, a series of conditions. The first one can be defined in abstract terms as the following property, to be satisfied by any pair of operators $A=A\left(t=t_{0}\right)$ and $B=B\left(t=t_{0}\right)$ (for some arbitrary value of $t_{0}$ ) evolved in some time parameter $t$ in the Heisenberg picture: there exists some $\beta \in \mathbb{R}$ such that

$$
\langle A(t-i \epsilon) B\rangle=\langle B A(t+i \beta-i \epsilon)\rangle .
$$

We have introduced a suitable regularization (the $i \epsilon$ terms) that is needed in order to formally manipulate distributions as functions. Translated in terms of the pullback of the Wightman function $W_{\ell}\left(\tau^{\prime \prime}, \tau^{\prime}\right)=\left\langle 0\left|\phi\left(X\left(\tau^{\prime \prime}\right)\right) \phi\left(X\left(\tau^{\prime}\right)\right)\right| 0\right\rangle$, the equation above reads

$$
W_{\ell}\left(\tau^{\prime \prime}-i \epsilon, \tau^{\prime}\right)=W_{\ell}\left(\tau^{\prime}, \tau^{\prime \prime}+i \beta-i \epsilon\right) .
$$

This explains why the KMS condition is sometimes defined in the literature (see, e.g., Refs. [31,42,52]) just as the symmetry of the pullback of the Wightman function under the transformation $\tau^{\prime \prime} \rightarrow \tau^{\prime}+i \epsilon$ and $\tau^{\prime} \rightarrow \tau^{\prime \prime}+i \beta-i \epsilon$, with $\beta=2 \pi / a$.

When Eq. (5) is satisfied by all the possible operators $A$ and $B$, including the field operators $\phi(X)$ but also the identity operator $\mathbb{1}$, it follows that the state $|0\rangle$ must be invariant under time translations. As a consequence, the pullback of the Wightman function must be stationary [51], namely, invariant under translations in $\tau$ or, equivalently, a function of $z$ only. Note that Eq. (6) being satisfied does not therefore imply by itself stationarity. This is especially important for the present discussion, as it is not difficult to show that the pullback to uniformly accelerated trajectories of any explicitly Lorentz-violating Wightman function cannot be stationary, and therefore it must necessarily violate the KMS condition (the stationarity condition can still be satisfied on inertial trajectories [7,9]).

However, the KMS condition involves additional restrictions [44,51], which are not always emphasized in the literature. The pullback of the Wightman function must be holomorphic in $z$ in a horizontal strip of the lower complex semiplane with a width $2 \pi / a$ in the $\epsilon \rightarrow 0$ limit, being the real axis one of its boundaries (under the condition of imaginary periodicity above, the position of this horizontal strip can be shifted by an arbitrary multiple of $2 \pi i / a$ ). Therefore, it is not only necessary to show that Eq. (6) holds, but also the absence of poles inside this horizontal strip must be shown in order to claim that the KMS 
condition holds (equivalently, any poles must be located in the boundary of the strip in the $\epsilon \rightarrow 0$ limit). Lastly, there is another further condition, which is similar but more restrictive than our requirement (3): the pullback of the Wightman function inside the complex strip must be polynomially bounded.

These are all the ingredients that are needed in order to compare the KMS condition with the definition for the preservation of the Unruh effect given in this Letter. This is summarized in Table I. We can see that, as anticipated in the introduction of this letter, the KMS condition is a sufficient but not necessary condition for the preservation of the Unruh effect. In particular, violating the imaginary periodicity is not sufficient in order to claim that the Unruh effect is not present. The second observation is about the interplay between the imaginary periodicity of the Wightman function and stationarity. It may seem surprising that there are deformations that satisfy the former but not the latter. However, as discussed above, stationarity follows from the stronger condition of imaginary periodicity for arbitrary pairs of operators (including the identity $\mathbb{1}$ ). Moreover, it is not difficult to see that this feature is quite general, as one can show that

$$
\begin{aligned}
& W_{\ell}\left(\tau^{\prime}, \tau^{\prime \prime}+i \beta-i \epsilon\right) \\
& \quad=\mathcal{W}_{\ell}\left(\Delta t\left(\tau^{\prime}, \tau^{\prime \prime}+i \beta-i \epsilon\right), \Delta \boldsymbol{x}\left(\tau^{\prime}, \tau^{\prime \prime}+i \beta-i \epsilon\right)\right) \\
& \quad=\mathcal{W}_{\ell}\left(-\Delta t\left(\tau^{\prime \prime}-i \epsilon, \tau^{\prime}\right),-\Delta \boldsymbol{x}\left(\tau^{\prime \prime}-i \epsilon, \tau^{\prime}\right)\right) .
\end{aligned}
$$

The first identity just makes explicit that the pullback of the Wightman function depends on $\tau^{\prime}$ and $\tau^{\prime \prime}$ implicitly through the time and space intervals, while the second identity exploits the periodicity properties of hyperbolic functions. It follows that any Wightman function that is even in the time and space intervals satisfies the imaginary periodicity condition, which is, moreover, equivalent to the symmetry under $z \rightarrow z+i \beta$.

Conclusions.-Exploiting a previously unnoticed symmetry of the Wightman function, we have been able to analyze in a general and systematic way the conditions that guarantee the appearance of the Unruh effect, understood as the thermal response of Unruh-DeWitt detectors. Our analysis settles the practical issue of determining whether or not a particular deformation of a QFT preserves the Unruh effect, providing the necessary tools to answer this question in a wide range of scenarios. As an application of our formalism, we have provided explicit examples in which the KMS condition is violated in different ways, while the response of a detector in the adiabatic limit still displays a thermal behavior. Thus, in these scenarios, no adiabatic thermalization experiment will find any contradiction with the Unruh effect even if the pullback of the vacuum two-point function is not KMS.

The authors thank Jorma Louko and Stefano Liberati for helpful discussions. This work has been supported in part by the MINECO (Spain) Projects No. FIS2016-78859-P and No. FIS2017-86497-C2-2 (with FEDER contribution). The work of E. M.-M. is supported by the Natural Sciences and Engineering Research Council of Canada through the Discovery program. E. M.-M. also gratefully acknowledges the funding of his Ontario Early Research Award. R. C.-R. gratefully acknowledges the hospitality of Perimeter Institute and the funding of his visit. This research was supported in part by Perimeter Institute for Theoretical Physics. Research at Perimeter Institute is supported by the Government of Canada through the Department of Innovation, Science and Economic Development and by the Province of Ontario through the Ministry of Research and Innovation.

*raul.carballorubio@sissa.it

†uisj.garay@ucm.es

*emartinmartinez@uwaterloo.ca

§jderamonrivera@uwaterloo.ca

[1] L. J. Garay, Quantum gravity and minimum length, Int. J. Mod. Phys. A 10, 145 (1995).

[2] J. Collins, A. Perez, D. Sudarsky, L. Urrutia, and H. Vucetich, Lorentz Invariance and Quantum Gravity: An Additional Fine-Tuning Problem? Phys. Rev. Lett. 93, 191301 (2004).

[3] J. Collins, A. Perez, and D. Sudarsky, Lorentz invariance violation and its role in quantum gravity phenomenology, arXiv:hep-th/0603002.

[4] R. Gambini, S. Rastgoo, and J. Pullin, Small Lorentz violations in quantum gravity: do they lead to unacceptably large effects? Classical Quantum Gravity 28, 155005 (2011).

[5] J. Polchinski, Comment on 'Small Lorentz violations in quantum gravity: do they lead to unacceptably large effects?' Classical Quantum Gravity 29, 088001 (2012).

[6] A. Belenchia, A. Gambassi, and S. Liberati, Lorentz violation naturalness revisited, J. High Energy Phys. 06 (2016) 049.

[7] V. Husain and J. Louko, Low Energy Lorentz Violation from Modified Dispersion at High Energies, Phys. Rev. Lett. 116, 061301 (2016).

[8] N. Kajuri, Polymer quantization predicts radiation in inertial frames, Classical Quantum Gravity 33, 055007 (2016).

[9] J. Louko and S. D. Upton, Low-energy Lorentz violation from high-energy modified dispersion in inertial and circular motion, Phys. Rev. D 97, 025008 (2018).

[10] N. Kajuri and G. Sardar, Low energy Lorentz violation in polymer quantization revisited, Phys. Lett. B 776, 412 (2018).

[11] A. Belenchia, D. M. T. Benincasa, E. Martín-Martínez, and M. Saravani, Low energy signatures of nonlocal field theories, Phys. Rev. D 94, 061902(R) (2016).

[12] A. Belenchia, D. M. T. Benincasa, S. Liberati, and E. Martín-Martínez, Transmission of information in nonlocal field theories, Phys. Rev. D 96, 116006 (2017).

[13] S. A. Fulling, Nonuniqueness of canonical field quantization in riemannian space-time, Phys. Rev. D 7, 2850 (1973). 
[14] P. C. W. Davies, Scalar production in Schwarzschild and Rindler metrics, J. Phys. A 8, 609 (1975).

[15] W. G. Unruh, Notes on black-hole evaporation, Phys. Rev. D 14, 870 (1976).

[16] L. C. B. Crispino, A. Higuchi, and G. E. A. Matsas, The Unruh effect and its applications, Rev. Mod. Phys. 80, 787 (2008).

[17] S. W. Hawking, Black hole explosions? Nature (London) 248, 30 (1974).

[18] S. W. Hawking, Particle creation by black holes, Commun. Math. Phys. 43, 199 (1975).

[19] R. Schutzhold, G. Schaller, and D. Habs, Tabletop Creation of Entangled Multi-keV Photon Pairs and the Unruh Effect, Phys. Rev. Lett. 100, 091301 (2008).

[20] M. Aspachs, G. Adesso, and I. Fuentes, Optimal Quantum Estimation of the Unruh-Hawking Effect, Phys. Rev. Lett. 105, 151301 (2010).

[21] E. Martín-Martínez, I. Fuentes, and R. B. Mann, Using Berry's Phase to Detect the Unruh Effect at Lower Accelerations, Phys. Rev. Lett. 107, 131301 (2011).

[22] E. Martín-Martínez, D. Aasen, and A. Kempf, Processing Quantum Information with Relativistic Motion of Atoms, Phys. Rev. Lett. 110, 160501 (2013).

[23] W. G. Unruh and R. M. Wald, What happens when an accelerating observer detects a Rindler particle, Phys. Rev. D 29, 1047 (1984).

[24] B. S. DeWitt, General Relativity: An Einstein Centenary Survey (Cambridge University Press, Cambridge, England, 1980), pp. 680-745.

[25] N. Sánchez, Quantum detection on the vacuum by nonuniformly accelerated observers, Phys. Lett. 105B, 375 (1981).

[26] P. G. Grove and A. C. Ottewill, Notes on 'particle detectors', J. Phys. A 16, 3905 (1983).

[27] N. D. Birrell and P.C. W. Davies, Quantum Fields in Curved Space, Cambridge Monographs on Mathematical Physics (Cambridge University Press, Cambridge, England, 1984).

[28] R. Kubo, Statistical-mechanical theory of irreversible processes. I. general theory and simple applications to magnetic and conduction problems, J. Phys. Soc. Jpn. 12, 570 (1957).

[29] P. C. Martin and J. Schwinger, Theory of many-particle systems. I, Phys. Rev. 115, 1342 (1959).

[30] R. Haag, N. M. Hugenholtz, and M. Winnink, On the equilibrium states in quantum statistical mechanics, Commun. Math. Phys. 5, 215 (1967).

[31] G. M. Hossain and G. Sardar, Violation of the Kubo-MartinSchwinger condition along a Rindler trajectory in polymer quantization, Phys. Rev. D 92, 024018 (2015).

[32] W. G. Brenna, E. G. Brown, R. B. Mann, and E. MartínMartínez, Universality and thermalization in the Unruh effect, Phys. Rev. D 88, 064031 (2013).
[33] J. Louko and D. Marolf, Inextendible Schwarzschild black hole with a single exterior: How thermal is the Hawking radiation? Phys. Rev. D 58, 024007 (1998).

[34] S. De Bievre and M. Merkli, The Unruh effect revisited, Classical Quantum Gravity 23, 6525 (2006).

[35] I. Agulló, J. Navarro-Salas, G. J. Olmo, and L. Parker, Twopoint functions with an invariant Planck scale and thermal effects, Phys. Rev. D 77, 124032 (2008).

[36] M. Rinaldi, Superluminal dispersion relations and the Unruh effect, Phys. Rev. D 77, 124029 (2008).

[37] P. Nicolini and M. Rinaldi, A minimal length versus the Unruh effect, Phys. Lett. B 695, 303 (2011).

[38] D. Campo, Problems with models of a fundamental length, arXiv:1004.5324.

[39] S. Gutti, S. Kulkarni, and L. Sriramkumar, Modified dispersion relations and the response of the rotating UnruhDeWitt detector, Phys. Rev. D 83, 064011 (2011).

[40] I. Agulló, J. Navarro-Salas, G. J. Olmo, and L. Parker, Acceleration radiation, transition probabilities and transPlanckian physics, New J. Phys. 12, 095017 (2010).

[41] G. M. Hossain and G. Sardar, Is there Unruh effect in polymer quantization? Classical Quantum Gravity 33, 245016 (2016).

[42] N. Alkofer, G. D’Odorico, F. Saueressig, and F. Versteegen, Quantum gravity signatures in the Unruh effect, Phys. Rev. D 94, 104055 (2016).

[43] A. S. Wightman, Quantum field theory in terms of vacuum expectation values, Phys. Rev. 101, 860 (1956).

[44] C. J. Fewster, B. A. Juárez-Aubry, and J. Louko, Waiting for Unruh, Classical Quantum Gravity 33, 165003 (2016).

[45] L. J. Garay, E. Martín-Martínez, and J. de Ramon, Thermalization of particle detectors: The Unruh effect and its reverse, Phys. Rev. D 94, 104048 (2016).

[46] S. Takagi, Vacuum noise and stress induced by uniform acceleration: Hawking-Unruh effect in rindler manifold of arbitrary dimension, Prog. Theor. Phys. Suppl. 88, 1 (1986).

[47] See Supplemental Material at http://link.aps.org/ supplemental/10.1103/PhysRevLett.123.041601 for the derivation of Eq. (3) using our conditions (1)-(3), as well as the discussion of other relevant mathematical identities.

[48] M. Rinaldi, Particle production and transplanckian problem on the non-commutative plane, Mod. Phys. Lett. A 25, 2805 (2010).

[49] P. Langlois, Causal particle detectors and topology, Ann. Phys. (Berlin) 321, 2027 (2006).

[50] C. Rovelli and M. Smerlak, Unruh effect without transhorizon entanglement, Phys. Rev. D 85, 124055 (2012).

[51] F. Strocchi, Thermal states, in Symmetry Breaking (Springer, Berlin, 2008), pp. 139-150.

[52] C. Rovelli, LQG predicts the Unruh Effect. Comment to the paper "Absence of Unruh effect in polymer quantization" by Hossain and Sardar, arXiv:1412.7827. 